\title{
Preface: A volume dedicated to Wolfgang Rump on the occasion of his 65th birthday
}

\author{
Yichuan Yang ${ }^{1}$
}

Published online: 29 March 2017

(C) Springer-Verlag Berlin Heidelberg 2017

\begin{abstract}
International Conference On Logical Algebras and Semirings - In honor of the 65th birthday of Wolfgang Rump Prof. Dr. Wolfgang Rump has played a leading role in the fields of partially ordered algebraic systems, semantics of non-commutative algebraic logic, and related quantum structures. As his 65 th birthday and his retirement from University of Stuttgart approached, the urge on the part of many to use this occasion for a conference became irresistible. Of course, Beijing is one of the obvious venues.
\end{abstract}

The conference on Logical Algebras and Semi-rings 2015 (LAS 2015 for short) was organized at the Haidian campus of Beihang University. Mathematicians from around the world came to share their work and to promote research across that broad range of mathematics that includes domain theory, quantum logic, Non-commutative logical algebras, lattice-ordered rings and fields, states on BCI-algebras, semigroups, accelerated modulus, semimodules, Lukasiewicz rings, semi-rings and their relatives, and the interaction of these fields with theoretic computer science.

With LAS 2015, our mathematical community recognized and celebrated the mathematical achievements on related topics of our friend and colleague Wolfgang Rump.

The present volume of Soft Computing largely consists of selected papers that arose in connection with LAS 2015.

Communicated by Y. Yang.

The conference is partially supported by NSFC (Grant 11271040), and the Fundamental Research Funds for the Central Universities (Grant 302996).

Yichuan Yang

ycyang@buaa.edu.cn

1 Department of Mathematics, Beihang University, Beijing 100191, China

\section{Life and research of Wolfgang Rump}

Wolfgang Rump was born in a small village near Wuppertal, five years after the end of the second world war, on October 16, 1950. His father Hubert, a merchant, stems from West Prussia, while his mother Elisabeth had an Italian father and a mother from an old German family. At the age of six, his parents moved to a town in the Palatinate region, where he went to school until his study in Kaiserslautern and Stuttgart. He spent his childhood by drawing trees, clouds, and flower girls, and by wondering about the ratio between circumference and diameter of a circle. His first love was Geometry, though for a while, his interests were focussed upon physics. His younger brother Siegfried could not escape to become a mathematician, too. Until 1975, they studied in the same university of Kaiserslautern. Siegfried went to applied mathematics and got his PhD in Karlsruhe. After a four years' engagement in IBM near Stuttgart, he became the head of the Institute for Reliable Computing at the University of Hamburg-Harburg.

Wolfgang sticked to pure mathematics. During a two year's military service, he was in correspondence with mathematicians from Erlangen-Nürnberg and Frankfurt, discussing questions about logic and topology. In Kaiserslautern he started with Integral Equations (B. Gramsch) and Functional Analysis (Klaus Bierstedt $\dagger$ ) before he became interested in algebraic topics through lectures and seminars of Kai Faltings. Before his move to Stuttgart in 1975, he learned to know H. J. Nastold, the supervisor of Gerd Faltings, at a summer school of the "Studienstiftung" on modular representation theory of finite groups. In 1977, he graduated under Klaus Roggenkamp with a prize-winning diploma thesis on representation theory of orders, the second part of which became his $\mathrm{PhD}$ thesis in 1978. In that year, Wolfgang met his future wife Ky Phan (Chinese) who followed him, eight months later, to Urbana (Illinois) and Columbus (Ohio) where he spent one year with a research grant by the German Research 
Council (DFG). In Ohio, he stayed with Hans Zassenhaus, one of the old hands in number theory and group theory. He used the morning hours for learning Chinese, to translate Chinese poems from Tang dynasty, and to acquire the skills for writing a marriage proposal to the parents of Ky Phan. In Illinois, he stayed with Irving Reiner, the "father" of integral representation theory and shared his office with Phillip Griffith next to that of Robert Fossum. After his return to Germany, he worked for two years at Siemens in Stuttgart and Munich, as a marketing consultant and system developer, using the access to big computers for calculations of indecomposable representations of Cohen-Macaulay orders. After his marriage in December 1980, it became obvious, that spending a whole day for earning money could not be a lasting perspective for a born mathematician. So, with the consent of his wife, he embarked on the rocky future of an uncompromising pure mathematician. He went to Eichstätt, a small university in the north of Bavaria, as an assistant of Jürgen Rohlfs, a student of Günter Harder. Mathematically, most people would not find much attraction in Eichstätt. For Wolfgang, nevertheless, the first impression was overwhelming. The famous town with its religious life and long history offered a great treasure and big compensation. A son was born in 1982, and the family lived there for 25 years.

The quiet place invited to contemplation. Superficially, that part of life could be seen as a long walk through the Middle Ages. Mathematics appeared as a tiny part of a much bigger cosmos of science, inexhaustible by human labor, and inevitably going beyond a man's lifetime.

The early work of Wolfgang Rump found admirers mostly in the eastern part of Europe. Many visits led him to conferences and lecture tours in Poland, Ukraine, Russia, and Romania. On the Fourth International Conference on Representation Theory of Algebras 1984 in Ottawa, he presented his four infinite series and 854 exceptional indecomposable representations of a class of tame orders, acclaimed by Pierre Gabriel, who inaugurated the new branch of quiver representations in 1970, and Andrei Vladimirovich Roiter, his Russian counterpart, whose "chess-board" approach to representations culminated in his invention of bocses. Wolfgang Rump's 1989 habilitation treatise gives a detailed account of this classification, including a stability theorem for representation-finite orders, which led to unexpected relationships between different types of orders. This 405 pages treatise is typical for his first long period of work, somehow similar to the Russian style of the Soviet era, remote from the main stream of western mathematics, with long unpublished manuscripts and few articles of moderate length in current mathematical journals. Daniel Simson, on a visit in Eichstätt once asked him whether he would translate the main results of his "bible" into readable mathematics in due course. He made a serious attempt to do so, but soon gave up by lack of time and limited readership.

According to MathSciNet, Wolfgang Rump has published 97 articles in mathematical journals, more than eighty percent after the millennium when he reached the age of fifty. Most of his papers deal with representation theory of associative rings and algebras, especially Cohen-Macaulay orders, Auslander-Reiten theory of orders in semisimple and non-semisimple algebras, large lattices, non-commutative algebraic geometry and arithmetic, categories and homological algebra, and quantum theory. Inspired by investigations of Igusa, Todorov, and Iyama, he developed a theory of "ladder functors", acting on morphisms instead of objects, which makes Auslander-Reiten theory functoral. Using ladder functors, he constructed large indecomposable representations of Cohen-Macaulay orders and characterized AuslanderReiten quivers of representation-finite algebras and orders in terms of additive functions. Other contributions to representation theory deal with differentiation of orders, quadratic forms, and vector space categories. He found a conceptual and most general form of Zavadskij's differentiation algorithm for categories of Krull dimension one. His paper on "almost abelian categories" led to several new developments in topological algebra, tilting theory, functional analysis, homological algebra, and Morita duality. In functional analysis, he worked on classes of barreled and bornological spaces which led to counter examples of an old conjecture of Raikov.

Since 2005 , he became interested in $\ell$-groups and quantum theory. He related algebraic logic to the quantum Yang-Baxter equation via $L$-algebras and cycle sets. Using cycle sets, he established Gateva-Ivanova's conjectured equivalence between square-free solutions and certain noncommutative regular rings. He introduced the concept of braces, providing a further connection to radical rings and nilpotency problems arising in non-commutative ring theory. His paper on $\mathbb{R}$-linear braces and classical groups led to a negative solution of the problem whether every finite solvable group is an involutive Yang-Baxter group. Quite recently, he introduced the concept of right $\ell$-group which relates Artin-Tits groups, knot groups, and other Garside groups arising in topology, as well as von-Neumann algebras and non-commutative number theory, to classical lattice-ordered groups.

During a later visit to Eichstätt, he wrote an essay (with R. Felix) on mathematics at a "catholic university" (in German). He wrote a book on Galois theory, a brief account of 120 pages (unpublished) with exercises, for the use of students to get a quick introduction without detours into this fascinating theory. Among his students are Steffen Oppermann, professor at NTNU in Trondheim, Simon Schmider who took a postdoctoral position in Eichstaett, and the author of this preface, Yichuan Yang, Professor 
at Beihang University in Beijing. Our joint work led to several papers on abelian $\ell$-groups, their spectral properties and relationship to Bézout domains, non-commutative logical algebras like pseudo-MV algebras and quantales, non-commutative arithmetic, and non-archimedean directed fields. Some papers deal with L-algebras and quantum Balgebras, a new development which greatly unifies many of these structures. 